\title{
NONSTATIONARITY AND RANDOMIZATION IN THE REED-FROST EPIDEMIC MODEL
}

\author{
CLAUDE LEFÈVRE, ${ }^{*}$ Université Libre de Bruxelles \\ PHILIPPE PICARD, ${ }^{* *}$ Université de Lyon 1
}

\begin{abstract}
The purpose of this paper is to determine the exact distribution of the final size of an epidemic for a wide class of models of susceptible-infective-removed type. First, a nonstationary version of the classical Reed-Frost model is constructed that allows us to incorporate, in particular, random levels of resistance to infection in the susceptibles. Then, a randomized version of this nonstationary model is considered in order to take into account random levels of infectiousness in the infectives. It is shown that, in both cases, the distribution of the final number of infected individuals can be obtained in terms of Abel-Gontcharoff polynomials. The new methodology followed also provides a unified approach to a number of recent works in the literature.
\end{abstract}

Keywords: Epidemic; final size; random susceptibility; vaccination; random infectivity; martingale; Abel-Gontcharoff polynomial

2000 Mathematics Subject Classification: Primary 60J80; 60K99

Secondary $92 \mathrm{D} 30$

\section{Introduction}

The Reed-Frost chain-binomial epidemic model is a classical model that describes the spread of infectious diseases of susceptible-infective-removed (SIR) type. It is concerned with a closed, homogeneous, and independently mixing population. Initially, the population contains $n$ susceptibles, $m$ infectives, and no removed cases. If contacted by an infective, an individual goes through a latent period of fixed length followed by an infectious period that is contracted to a single point of time. At that instant, the infective is able to contact any given susceptible with probability $p=1-q, 0<q<1$, all possible encounters being independent of each other. The infective then recovers and becomes immune to the disease, so being removed from the infection process.

We take a discrete time scale $t=0,1,2, \ldots$, to represent the successive generations of infectives. If at time $t$ there are $I_{t}$ infectives, any of the $S_{t}$ susceptibles will remain susceptible at time $t+1$ with probability $q^{I_{t}}$. Thus, the conditional law of $S_{t+1}$ is of binomial type, i.e.

$$
S_{t+1} \mid\left(S_{t}, I_{t}\right) \stackrel{\mathrm{D}}{=} \operatorname{bin}\left[S_{t}, q^{I_{t}}\right], \quad t \in \mathbb{N},
$$

where $S_{0}=n, I_{0}=m$, and $\mathbb{N}=\{0,1,2, \ldots\}$. Moreover, $I_{t+1}=S_{t}-S_{t+1}$ by construction. The epidemic ceases as soon as there are no more infectives in the population, which happens

Received 4 March 2005; revision received 16 July 2005.

* Postal address: Institut de Statistique et de Recherche Opérationnelle, CP 210, Université Libre de Bruxelles, Boulevard du Triomphe, B-1050 Bruxelles, Belgium. Email address: clefevre@ulb.ac.be

** Postal address: Institut de Science Financière et d'Assurances, Université de Lyon 1, 50 Avenue Tony Garnier, F-69007 Lyon, France. 
almost surely after a finite time $T$. Hence, $S_{T}$ gives the ultimate number of susceptibles escaping the disease and $F_{T}=n-S_{T}$ is the total number of newly infected cases.

The statistics $S_{T}$ and $F_{T}$ play an important role in theory and practice. Their study, exact and asymptotic, has received considerable attention in the literature. Hereafter, we will focus on the determination of their exact distributions. As shown in several works (e.g. von Bahr and Martin-Löf (1980) and Lefèvre and Picard (1990)), the law of $S_{T}$ satisfies the $n$ relations

$$
\mathrm{E}\left\{S_{T,[k]} q^{k S_{T}}\right\}=n_{[k]} q^{k(m+n)}, \quad k=1, \ldots, n,
$$

using the notation $x_{[k]}=x(x-1) \cdots(x-k+1)$, for any natural numbers $x$ and $k$. The system (1.2) provides $n$ linear equations in the $n$ unknown probabilities $\mathrm{P}\left(S_{T}=s\right), s=1, \ldots, n$. It can be solved recursively for $k=n, \ldots, 1$, and $\mathrm{P}\left(S_{T}=0\right)$ then follows.

Relations (1.2) can be exploited to derive a (quasi)explicit formula for the law of $F_{T}$ in terms of remarkable polynomials named Abel-Gontcharoff polynomials. More precisely, let $U=\left\{u_{i}, i \in \mathbb{N}\right\}$ be a given sequence of real numbers. Denote by $G_{j}(x \mid U), j \in \mathbb{N}$, the unique family of Abel-Gontcharoff polynomials of degree $j$ in $x$ associated with $U$. For a definition and basic properties, we refer the reader to the brief outline given in Appendix A. Lefèvre and Picard (1990) proved from (1.2) that

$$
\mathrm{P}\left(F_{T}=j\right)=n_{[j]} q^{(m+j)(n-j)} G_{j}\left(1 \mid\left\{q^{m+i}, i \in \mathbb{N}\right\}\right), \quad j=0, \ldots, n .
$$

This elegant formula illustrates the algebraic structure underlying the distribution of $F_{T}$. It is also of practical interest, since the Abel-Gontcharoff polynomials can be numerically determined by means of efficient recursive methods.

Let us recall that $G_{j}(x \mid U), j \geq 1$, depends on $U$ only through its first $j$ elements, and that $G_{0}(x \mid U)=1$. Thus, for instance, for each $G_{j}, 1 \leq j \leq n$, of (1.3), the set of indices $i \in \mathbb{N}$ may be replaced by $i, 0 \leq i \leq j-1$. In the sequel, however, in such a case it will be more convenient to write $0 \leq i \leq n$ for each $G_{j}, 0 \leq j \leq n$.

The Reed-Frost model relies on rather specific assumptions, and a number of variants and generalizations have been proposed for infectious diseases of SIR type. Much on this can be found in the recent comprehensive treatises by Daley and Gani (1999) and Andersson and Britton (2000); see also the review paper by Lefèvre (1990).

Our purpose in the present paper is to point out how formulae (1.2) and (1.3) can be extended to incorporate nonstationarity in the individual contact probabilities. Such a generalization allows us to cover, in particular, situations in which the susceptibles display random levels of susceptibility (this arises, for instance, in a vaccination context when vaccine response is random). Furthermore, a randomization of the contact probabilities in the nonstationary model will then allow us to deal with situations in which the infectives exhibit random levels of infectiousness (this is usually due to the variability of the length of the infectious periods).

To the best of the authors' knowledge, a nonstationary Reed-Frost model has not yet been discussed. Nevertheless, the case of random infectiousness has been widely investigated (it is generally called the randomized Reed-Frost model); see, for instance, Ludwig (1975), von Bahr and Martin-Löf (1980), Ball (1986), Martin-Löf (1986), and Picard and Lefèvre (1990). The case of random susceptibility is less studied; see, for instance, Ludwig (1975), Picard and Lefèvre (1991), and a similar model for vaccination of Becker and Utev (2002). Combining both random infectiousness and susceptibility generates additional, often considerable, difficulties; see, for instance, Scalia-Tomba (1985) and Ball and O’Neill (1999). 


\section{Nonstationary version of the model}

\subsection{Final state distribution}

To construct a nonstationary Reed-Frost model, we first assign to each infective - initial or newly infected - a label $l$ taking values in $1,2, \ldots, m+n$. For clarity, we here interpret $l$ as specifying a (possibly arbitrary) order of infection of the infectives over the course of time. Then, with each infective $l$, we associate a probability $p_{l}=1-q_{l}, 0<q_{l}<1$, that represents the probability of this infective transmitting the disease to any given susceptible still present at that time. Note that, since $l$ indicates an order of infection, each susceptible still present has necessarily escaped contact with the infectives labelled $1, \ldots, l-1$. Thus, $p_{l}$ in fact corresponds to the conditional probability that a susceptible is contacted by infective $l$ given that it has escaped infection by the previous $l-1$ infectives. Obviously, $p_{m+n}$ will have no role in the analysis (since there are no more susceptibles when all $m+n$ individuals have been infected); to unify the presentation, however, we prefer not to neglect it. Encounters made by different infectives are still assumed to be independent.

We are in a position to generalize the binomial formulation (1.1). For the first generation of infectives,

$$
S_{1} \mid\left(S_{0}=n, I_{0}=m\right) \stackrel{\mathrm{D}}{=} \operatorname{bin}\left[n, \prod_{l=1}^{m} q_{l}\right] .
$$

More generally, until generation $t \in \mathbb{N}$, the total number of infected cases is equal to $m+n-S_{t}$. Thus, the $I_{t}$ infectives present in generation $t$ are labelled as $m+n-S_{t}-I_{t}+1, \ldots, m+n-S_{t}$. For the conditional laws, we then obtain

$$
S_{t+1} \mid\left(S_{t}, I_{t}\right) \stackrel{\mathrm{D}}{=} \operatorname{bin}\left[S_{t}, \prod_{l=m+n-S_{t}-I_{t}+1}^{m+n-S_{t}} q_{l}\right], \quad t \in \mathbb{N} .
$$

To work with an expression simpler than (2.1), we introduce the parameters $\pi_{l}=q_{1} \cdots q_{l}$, $l=1, \ldots, m+n$. Obviously, $\pi_{l}$ represents the probability that a susceptible escapes contact with the first $l$ infectives. Throughout the paper, we set $\pi_{0}=1$. Then (2.1) becomes

$$
S_{t+1} \mid\left(S_{t}, I_{t}\right) \stackrel{\mathrm{D}}{=} \operatorname{bin}\left[S_{t}, \frac{\pi_{m+n-S_{t}}}{\pi_{m+n-S_{t}-I_{t}}}\right], \quad t \in \mathbb{N} .
$$

Since $I_{t}=S_{t-1}-S_{t}$ for $t \geq 1$, and holds too for $t=0$ when defining $S_{-1}=m+n$, we can finally rewrite (2.2) as

$$
S_{t+1} \mid \mathcal{F}_{t} \stackrel{\mathrm{D}}{=} \operatorname{bin}\left[S_{t}, \frac{\pi_{m+n-S_{t}}}{\pi_{m+n-S_{t-1}}}\right], \quad t \in \mathbb{N},
$$

where $\mathcal{F}_{t}$ denotes the $\sigma$-field generated by $\left\{S_{-1}, \ldots, S_{t}\right\}$. Note that, for the classical Reed-Frost model, $q_{l}=q$ and $\pi_{l}=q^{l}$ for each $l$, meaning that (2.3) reduces to (1.1).

Let $T$ represent the time at which the epidemic ends. We will show that the distributions of $S_{T}$ and $F_{T}$ are provided by formulae (2.4) and (2.5) below, which generalize the previous results (1.2) and (1.3).

Proposition 2.1. We have

$$
\mathrm{E}\left\{\frac{S_{T,[k]}}{\left(\pi_{\left.m+n-S_{T}\right)^{k}}\right.}\right\}=n_{[k]}, \quad k=1, \ldots, n,
$$


yielding

$$
\mathrm{P}\left(F_{T}=j\right)=n_{[j]}\left(\pi_{m+j}\right)^{n-j} G_{j}\left(1 \mid\left\{\pi_{m+i}, 0 \leq i \leq n\right\}\right), \quad j=0, \ldots, n .
$$

Proof. From (2.3), the conditional factorial moments of $S_{t+1}$ are given by

$$
\mathrm{E}\left\{S_{t+1,[k]} \mid \mathcal{F}_{t}\right\}=S_{t,[k]}\left(\frac{\pi_{m+n-S_{t}}}{\pi_{m+n-S_{t-1}}}\right)^{k}, \quad k=1, \ldots, n .
$$

Thus,

$$
\left\{\frac{S_{t,[k]}}{\left(\pi_{m+n-S_{t-1}}\right)^{k}}, \mathcal{F}_{t}, t \in \mathbb{N}\right\}, \quad k=1, \ldots, n, \quad \text { is a martingale. }
$$

By definition, $T$ is the first time $t \geq 1$ such that $I_{t}=0$, i.e. $S_{t}=S_{t-1}$. Obviously, it corresponds to a stopping time for the martingales (2.6). By applying the optional stopping theorem, we then obtain the $n$ relations (2.4).

We now notice that

$$
\left(\begin{array}{l}
s \\
k
\end{array}\right)\left(\begin{array}{l}
n \\
k
\end{array}\right)^{-1}=\left(\begin{array}{l}
n-k \\
n-s
\end{array}\right)\left(\begin{array}{c}
n \\
n-s
\end{array}\right)^{-1},
$$

for any natural numbers $k, s$, and $n, 0 \leq k \leq s \leq n$. Since $n-S_{T}=F_{T},(2.4)$ is equivalent to

$$
\mathrm{E}\left\{\frac{\left(\begin{array}{c}
n-k \\
F_{T}
\end{array}\right)}{\left(\begin{array}{c}
n \\
F_{T}
\end{array}\right)\left(\pi_{m+F_{T}}\right)^{k}}\right\}=1, \quad k=1, \ldots, n,
$$

which identity also holds for $k=0$. More explicitly, this yields

$$
\sum_{j=0}^{n-k}(n-k)_{[j]}\left(\pi_{m+j}\right)^{n-k-j} a_{j}=1, \quad k=0, \ldots, n,
$$

where

$$
a_{j}=\frac{\mathrm{P}\left(F_{T}=j\right)}{n_{[j]}\left(\pi_{m+j}\right)^{n-j}}, \quad j=0, \ldots, n .
$$

It remains to observe that (2.7) can be identified with the Abelian expansion (A.3), below, of the polynomial $x^{n-k}$ constructed from the family $U=\left\{u_{i}=\pi_{m+i}, 0 \leq i \leq n\right\}$ and evaluated at $x=1$. Therefore, $a_{j}=G_{j}\left(1 \mid\left\{\pi_{m+i}, 0 \leq i \leq n\right\}\right)$ for $0 \leq j \leq n$, from which we recover (2.5).

Remark 2.1. Let us generalize the model by allowing susceptibles also to be infected from outside the population (as, for instance, in Addy et al. (1991) and Ball et al. (1997)). Specifically, suppose that each of the $n$ susceptibles has probability $p_{0}=1-q_{0}$ of being infected by an external source of infection during the course of the epidemic, independently of the other members of the population.

The final size in such a model is easily seen to have the same distribution as that of the model without infection from outside, but now with random initial sizes $\left(S_{0}, I_{0}\right)$, where $S_{0} \stackrel{\mathrm{D}}{=} \operatorname{bin}\left[n, q_{0}\right]$ and $I_{0} \stackrel{\mathrm{D}}{=} m+\operatorname{bin}\left[n, p_{0}\right]$ (whence $S_{0}+I_{0}=m+n$, as before). We then observe that (2.6) remains valid, meaning that the optional stopping theorem gives

$$
\mathrm{E}\left\{\frac{S_{T,[k]}}{\left(\pi_{m+n-S_{T}}\right)^{k}}\right\}=n_{[k]} q_{0}^{k}, \quad k=1, \ldots, n,
$$


instead of (2.4). Moreover, straightforward adaptations of (2.7) and (2.8) then yield

$$
\mathrm{P}\left(F_{T}=j\right)=n_{[j]}\left(q_{0} \pi_{m+j}\right)^{n-j} G_{j}\left(1 \mid\left\{q_{0} \pi_{m+i}, 0 \leq i \leq n\right\}\right), \quad j=0, \ldots, n,
$$

instead of (2.5). Note that if only external infection is possible (i.e. $\pi_{l}=1$ for each $l$ ), then $F_{T} \stackrel{\mathrm{D}}{=} \operatorname{bin}\left[n, p_{0}\right]$, as expected.

It is intuitively clear that the epidemic will be less severe if the probabilities of noninfection are large. This can be formalized as follows. Let $\hat{F}_{T}$ be the final number of new cases for a model with parameters $\hat{\pi}_{l}$, and denote by ' $\leq_{\mathrm{D}}$ ' the distributional order relation.

Property 2.1. If $\hat{\pi}_{l} \geq \pi_{l}$ for $1 \leq l \leq m+n$, then $\hat{F}_{T} \leq_{\mathrm{D}} F_{T}$.

Proof. By (2.5), the distribution function of $F_{T}$ is

$$
\mathrm{P}\left(F_{T} \leq k\right)=\sum_{j=0}^{k} n_{[j]}\left(\pi_{m+j}\right)^{n-j} G_{j}\left(1 \mid\left\{\pi_{m+i}, 0 \leq i \leq n\right\}\right), \quad k=0, \ldots, n,
$$

which corresponds, by (A.3), to the first $k$ terms in the Abelian expansion of $x^{n}$ constructed from $U=\left\{u_{i}=\pi_{m+i}, 0 \leq i \leq n\right\}$ and evaluated at $x=1$. Thus, to obtain the result it suffices to apply a comparison result given in Property 4.2 of Lefèvre and Picard (1993).

This property could be proved by other methods (coupling, for example). Moreover, we mention that it is possible to derive bounds on $\mathrm{P}\left(F_{T}=j\right)$ by bounding $G_{j}$ in (2.5), but this is omitted here.

\subsection{Application to vaccination}

In the framework of the classical Reed-Frost model, suppose that the population has been vaccinated against the disease and that the vaccine response varies randomly between individuals (such as discussed by Becker and Utev (2002)). More precisely, we assume that any given susceptible, if ever contacted by an infective (which occurs with probability $p=1-q$ ), can resist infection with a certain (personal) random probability, and that these resistance probabilities are independent, identically distributed random variables, distributed as $\varepsilon$, say.

It is clear that this model with vaccination corresponds to a particular case of the previous nonstationary Reed-Frost version in which

$$
\pi_{l}=\mathrm{E}\left\{(q+p \varepsilon)^{l}\right\}, \quad l=1, \ldots, m+n .
$$

Therefore, the final state distribution is provided by Proposition 2.1 written in terms of these probabilities $\pi_{l}$.

In the literature, special attention is paid to two particular kinds of protective effect for a vaccine (see, e.g. Smith et al. (1984))

(i) The vaccine confers either complete or zero protection, i.e. $\varepsilon=1$ with probability $\gamma_{1}$ and $\varepsilon=0$ with probability $\gamma_{0}=1-\gamma_{1}$. Such models are called all/nothing models. In this case, (2.9) yields $\pi_{l}=\gamma_{1}+\gamma_{0} q^{l}$ for all $l$, and (2.5) becomes

$$
\mathrm{P}\left(F_{T}=j\right)=n_{[j]} \gamma_{0}^{j}\left(\gamma_{1}+\gamma_{0} q^{m+j}\right)^{n-j} G_{j}\left(1 \mid\left\{q^{m+i}, 0 \leq i \leq n\right\}\right), \quad j=0, \ldots, n,
$$

after (A.4), below, has been used to simplify $G_{j}$. 
(ii) The vaccine confers partial, uniform protection, i.e. $\varepsilon=r$ almost surely, with $r$ fixed in $(0,1)$. Such models are called leaky models. In this case, $(2.9)$ reduces to $\pi_{l}=(q+p r)^{l}$ for all $l$, and we obtain a Reed-Frost model with parameter $q+\operatorname{pr}($ rather than $q$ ).

Let us return to the general situation (2.9). The protective efficacy of a vaccine is usually measured by the parameter $E\{\varepsilon\}$. Thus, it can be useful to estimate the distribution of $F_{T}$ in terms of this parameter. This is done below by deriving lower and upper bounds in the distributional sense. We emphasize that a result of a similar nature has been established by Becker and Utev (2002).

Property 2.2. Under (2.9), we have $F_{T}^{(1)} \leq_{\mathrm{D}} F_{T} \leq_{\mathrm{D}} F_{T}^{(2)}$, where $F_{T}^{(1)}$ is the final number of infections in an all/nothing model with parameters $\pi_{l}^{(1)}=\mathrm{E}\{\varepsilon\}+[1-\mathrm{E}\{\varepsilon\}] q^{l}, 1 \leq l \leq m+n$, and $F_{T}^{(2)}$ is the final number of infections in a leaky model with parameters $\pi_{l}^{(2)}=[q+\bar{p} \mathrm{E}\{\varepsilon\}]^{l}$, $1 \leq l \leq m+n$.

Proof. Let $g(\varepsilon)=(q+p \varepsilon)^{l}$, such that $\pi_{l}=\mathrm{E}\{g(\varepsilon)\}$. By convexity of the function $g(\cdot)$, we obtain the inequality

$$
[q+p \mathrm{E}\{\varepsilon\}]^{l} \leq \mathrm{E}\{g(\varepsilon)\} \leq \mathrm{E}\{\varepsilon\}+[1-\mathrm{E}\{\varepsilon\}] q^{l}, \quad l=1, \ldots, m+n .
$$

Indeed, the lower bound in (2.11) follows from Jensen's inequality. Furthermore, $g(\varepsilon) \leq$ $g(0)(1-\varepsilon)+g(1) \varepsilon$ and taking expectations yields the upper bound in (2.11). The result then follows directly from Property 2.1 .

The approximating model that yields the upper bound $F_{T}^{(2)}$ confers a partial and uniform protection with efficacy level $\mathrm{E}\{\varepsilon\}$. This comparison result thus means that replacing the random resistance level $\varepsilon$ by its mean $\mathrm{E}\{\varepsilon\}$ leads to a standard Reed-Frost model that will overestimate the final severity of the disease. On the other hand, the approximating model that yields the lower bound $F_{T}^{(1)}$ confers either complete or zero protection with probability of success $\mathrm{E}\{\varepsilon\}$. In other words, the lower bound $F_{T}^{(1)}$ is attained when the resistance level has a Bernoulli distribution with parameter $\mathrm{E}\{\varepsilon\}$.

\subsection{Application to randomized susceptibilities}

More generally, the nonstationary extension enables us to incorporate random levels of susceptibility. Thus, instead of (2.9), we could choose $\pi_{l}=\mathrm{E}\left\{R^{l}\right\}$ for $1 \leq l \leq m+n$, where $R$ is a random variable valued in $[0,1]$ that represents a global level of resistance to infection per susceptible. For instance, if $R$ is uniform on $[0,1]$ then $\pi_{l}=1 /(l+1)$.

A more natural way to introduce variable susceptibility in the Reed-Frost model is through the concept of random infection tolerance (such as discussed by Sellke (1983)). Specifically, suppose that each infective has a fixed power of infection, given by $d$, and that any given susceptible becomes infected when its total absorbed exposure to infection reaches a certain (personal) random level, distributed as $\xi$, say (with the usual independence assumptions); then,

$$
\pi_{l}=\mathrm{P}(\xi>l d), \quad l=1, \ldots, m+n .
$$

The authors were rather surprised to obtain a formula as simple as (2.5) for the model with randomized susceptibilities. Indeed, in a previous work on this model, Picard and Lefèvre (1991) derived a formula for $\mathrm{P}\left(F_{T}=j\right)$ that is much more complicated. The equivalence between the formulae, however, is established in Appendix A. 
Returning to (2.12), we see that when $\xi$ has an exponential distribution with parameter $\beta$, the model reduces to a Reed-Frost model with parameter $q=\exp (-\beta)$. Now consider the case in which $\xi$ has a uniform law on an interval $(0, \alpha)$, which yields

$$
\pi_{l}=1-l d / \alpha, \quad l=1, \ldots, m+n,
$$

with the constraint $\alpha \geq(m+n) d$ to guarantee the positivity of the $\pi_{l}$. We notice that the assumption of a uniform tolerance could be rather restrictive in an epidemic context. Let $\bar{d}=d / \alpha$.

Corollary 2.1. Under (2.13),

$$
\mathrm{P}\left(F_{T}=j\right)=\left(\begin{array}{c}
n \\
j
\end{array}\right) m \bar{d}[(m+j) \bar{d}]^{j-1}[1-(m+j) \bar{d}]^{n-j}, \quad j=0, \ldots, n .
$$

Proof. By (2.13), the polynomials $G_{j}$ in (2.5) are constructed from a family $U=\left\{\pi_{m+i}\right.$, $0 \leq i \leq n\}$ that is affine in $i$. As we recall in Appendix A, the $G_{j}$ in this case reduce to the standard Abel polynomials, i.e. by (A.6),

$$
G_{j}(1 \mid\{1-(m+i) \bar{d}, 0 \leq i \leq n\})=\frac{m \bar{d}[(m+j) \bar{d}]^{j-1}}{j !}, \quad j=0, \ldots, n .
$$

Substituting (2.15) into (2.5) then yields (2.14).

The law (2.14) is recognized to be a quasibinomial distribution of type I (see Consul (1974)). It is somewhat unexpected to obtain a completely explicit formula for the distribution of the final size in an SIR epidemic model. Recently, the authors found that this formula, with $\bar{d}=1 /(m+n)$, was derived by Islam et al. (1996, Equation (5)) using a random graph representation of the same model (in a different formulation). We point out that, in the case in which $\bar{d}=1 /(m+n)$, the insertion of (2.13) into (2.2) yields

$$
S_{t+1} \mid\left(S_{t}, I_{t}\right) \stackrel{\mathrm{D}}{=} \operatorname{bin}\left[S_{t}, S_{t} /\left(S_{t}+I_{t}\right)\right]
$$

which means that each susceptible meets, per unit of time, a single individual taken at random from the whole population (including itself).

\section{Randomized version of the model}

\subsection{Final state distribution}

Let us now examine a generalization of the Reed-Frost model that incorporates random levels of infectiousness. More precisely, each infective $l$ is assumed to contact any given susceptible still present with a random probability $P_{l}=1-Q_{l}$, and the $Q_{l}, 1 \leq l \leq m+n$, are independent, identically distributed random variables distributed as $Q$, say. Such a situation occurs when the infectious periods $D_{l}$ are of random length (instead of being instantaneous) and correspond to independent, identically distributed random variables distributed as $D$. For instance, if any pair of individuals can meet at the points of a Poisson process of rate $\beta$, then $Q_{l}=\exp \left(-\beta D_{l}\right)$. As indicated in the introduction, this extended model has received much attention in the literature.

Clearly, the model can be studied by randomizing the previous nonstationary version. We let $\Pi_{l}=Q_{1} Q_{2} \cdots Q_{l}, 1 \leq l \leq m+n$. Taking the expectation in (2.5), we then obtain

$$
\mathrm{P}\left(F_{T}=j\right)=n_{[j]} \mathrm{E}\left\{\left(\Pi_{m+j}\right)^{n-j} G_{j}\left(1 \mid\left\{\Pi_{m+i}, 0 \leq i \leq n\right\}\right)\right\}, \quad j=0, \ldots, n .
$$


In practice, it is not easy to determine the law of $F_{T}$ by means of (3.1). However, we are going to deduce an equivalent formula, given in (3.2) below, that is expressed solely in terms of the parameters $q(l)=\mathrm{E}\left\{Q^{l}\right\}, 0 \leq l \leq n$. This simplification provides a real advantage in numerical computations. We mention that (3.2) was derived in Picard and Lefèvre (1990), but by a method that does not rely on the nonstationary model.

Proposition 3.1. A simpler form for (3.1) is

$\mathrm{P}\left(F_{T}=j\right)=n_{[j]} \sum_{k=0}^{j} \frac{1}{k !}[q(n-k)]^{m+k} G_{j-k}(0 \mid\{q(n-j+i), 0 \leq i \leq j\}), \quad j=0, \ldots, n$.

Proof. We first expand $G_{j}$ in (3.1) using the standard Taylor expansion around 0; by (A.5), we obtain

$\mathrm{P}\left(F_{T}=j\right)=n_{[j]} \sum_{k=0}^{j} \frac{1}{k !} \mathrm{E}\left\{\left(\Pi_{m+j}\right)^{n-j} G_{j-k}\left(0 \mid\left\{\Pi_{m+k+i}, 0 \leq i \leq n-k\right\}\right)\right\}, \quad j=0, \ldots, n$.

For $0 \leq k \leq n$, let $\Pi_{0}^{(k)}=1$ and $\Pi_{l}^{(k)}=Q_{k+1} Q_{k+2} \cdots Q_{k+l}, 1 \leq l \leq m+n-k$. In (3.3), we can then write $\Pi_{m+j}=\Pi_{m+k} \Pi_{j-k}^{(m+k)}$ and $\Pi_{m+k+i}=\Pi_{m+k} \Pi_{i}^{(m+k)}$. By substituting these into (3.3) and then applying (A.4) to $G_{j-k}$, we obtain

$$
\mathrm{P}\left(F_{T}=j\right)=n_{[j]} \sum_{k=0}^{j} \frac{1}{k !} \mathrm{E}\left\{\left(\Pi_{m+k}\right)^{n-k}\left(\Pi_{j-k}^{(m+k)}\right)^{n-j} G_{j-k}\left(0 \mid\left\{\Pi_{i}^{(m+k)}, 0 \leq i \leq n-k\right\}\right)\right\} .
$$

However, each random variable $\Pi_{m+k}$ is independent of the random variables $\Pi_{i}^{(m+k)}, 0 \leq$ $i \leq n-k$, meaning that, by definition of $q(l)$, (3.4) becomes

$$
\mathrm{P}\left(F_{T}=j\right)=n_{[j]} \sum_{k=0}^{j} \frac{1}{k !}[q(n-k)]^{m+k} \mathrm{E}\left\{\left(\Pi_{j-k}^{(m+k)}\right)^{n-j} G_{j-k}\left(0 \mid\left\{\Pi_{i}^{(m+k)}, 0 \leq i \leq n-k\right\}\right)\right\} .
$$

To evaluate the expectation in (3.5), we have recourse to Theorem 4.1 of Picard and Lefèvre (2003), which gives the identity

$$
\mathrm{E}\left\{\left(\Pi_{j-k}^{(m+k)}\right)^{n-j} G_{j-k}\left(0 \mid\left\{\Pi_{i}^{(m+k)}, 0 \leq i \leq n-k\right\}\right)\right\}=G_{j-k}(0 \mid\{q(n-j+i), 0 \leq i \leq j\}) .
$$

Inserting (3.6) into (3.5) then yields (3.2).

From (3.1) and Property 2.1, we see that an increase in the $\Pi_{l}$, in a distributional sense (by decreasing $D$ for example), will lead to a less severe epidemic in the same sense. More flexible comparisons in terms of the parameters $q(l)$ were provided in Lefèvre and Utev (1996). For instance, respectively denote by ' $\leq$ icx' and ' $\leq_{\text {icv' }}$ ' the increasing-convex and increasing-concave order relations. If $Q \leq_{\text {icx }} \hat{Q}$ then the corresponding final numbers of infectives, $F_{T}$ and $\hat{F}_{T}$, are such that $F_{T} \geq_{\text {icv }} \hat{F}_{T}$. In particular, approximating the infec-

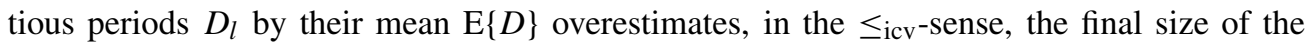
epidemic. 


\subsection{Combination with vaccination}

Let us re-examine the problem of vaccination discussed in Section 2.2 within the framework of the model presented above. Each infective $l$ contacts any given susceptible with a random probability $P_{l}=1-Q_{l}$, the $Q_{l}, 1 \leq l \leq m+n$, being independent, identically distributed random variables distributed as $Q$. In addition, if ever contacted, each susceptible escapes infection with a certain (personal) random probability distributed as $\varepsilon$, independently of the others. Thus, conditionally upon fixed values $p_{l}=1-q_{l}$ for the variables $P_{l}$, the model corresponds to a nonstationary Reed-Frost version in which the parameters are

$$
\pi_{l}^{c}=\mathrm{E}\left\{\prod_{l^{\prime}=1}^{l}\left(q_{l^{\prime}}+p_{l^{\prime}} \varepsilon\right)\right\}, \quad l=1, \ldots, m+n .
$$

The distribution of $F_{T}$ for this model can be determined by inserting (3.7) into (2.5) and then taking the expectation with respect to the variables $Q_{l}$ (at this stage, the $Q_{l}$ need not be identically distributed). Unfortunately, the evaluation of this expectation is, in general, very difficult. It is therefore of great interest to obtain lower and upper bounds that can be effectively calculated.

For that, we will again have recourse to the two special kinds of protective vaccine effect considered in Section 2.2.

(i) For partial, uniform protection, i.e. if $\varepsilon=r$ almost surely, (3.7) becomes

$$
\pi_{l}^{c}=\prod_{l^{\prime}=1}^{l}\left[r+(1-r) q_{l^{\prime}}\right], \quad l=1, \ldots, m+n .
$$

Thus, this leaky model is equivalent to the case of randomized infectiousness only in which, for each $l$, the probability $r+(1-r) Q_{l}$ is substituted for $Q_{l}$, as in Section 3.1. Consequently, the distribution of $F_{T}$ is given by (3.2) with, now,

$$
q(l)=\mathrm{E}\left\{[r+(1-r) Q]^{l}\right\}, \quad l=0, \ldots, n .
$$

(ii) For complete or zero protection, i.e. if $\varepsilon=1$ or 0 with respective probabilities $\gamma_{1}$ and $\gamma_{0}$, (3.7) yields

$$
\pi_{l}^{c}=\gamma_{1}+\gamma_{0} \prod_{l^{\prime}=1}^{l} q_{l^{\prime}}, \quad l=1, \ldots, m+n .
$$

In this all/nothing scheme, the distribution of $F_{T}$ is given by the simple formula (3.10) below. Note that (3.10) is rather intuitive and generalizes (2.10).

Corollary 3.1. Under (3.9),

$$
\mathrm{P}\left(F_{T}=j\right)=\sum_{k^{\prime}=0}^{n-j}\left(\begin{array}{l}
n \\
k^{\prime}
\end{array}\right)\left(\gamma_{0}\right)^{n-k^{\prime}}\left(\gamma_{1}\right)^{k^{\prime}} \mathrm{P}\left(F_{n-k^{\prime}, T}=j\right), \quad j=0, \ldots, n .
$$

Here, $F_{n-k^{\prime}, T}$ is the final number of infections in a model with randomized infectiousness only in which there are initially $n-k^{\prime}$ susceptibles and $m$ infectives and the contact probabilities are $P_{l}=1-Q_{l}$; its law is given by (3.2) with $n-k^{\prime}$ substituted for $n$. 
Proof. Instead of (3.1), we now have, for $j=0, \ldots, n$,

$$
\mathrm{P}\left(F_{T}=j\right)=n_{[j]} \mathrm{E}\left\{\left(\gamma_{1}+\gamma_{0} \Pi_{m+j}\right)^{n-j} G_{j}\left(1 \mid\left\{\gamma_{1}+\gamma_{0} \Pi_{m+i}, 0 \leq i \leq n\right\}\right)\right\},
$$

where $\Pi_{l}=Q_{1} Q_{2} \cdots Q_{l}$ for all $l$. By using (A.4) and expanding the factor $\left(\gamma_{1}+\gamma_{0} \Pi_{m+j}\right)^{n-j}$, we obtain

$\mathrm{P}\left(F_{T}=j\right)=\sum_{k^{\prime}=0}^{n-j}\left(\begin{array}{l}n \\ k^{\prime}\end{array}\right)\left(\gamma_{0}\right)^{n-k^{\prime}}\left(\gamma_{1}\right)^{k^{\prime}}\left(n-k^{\prime}\right)_{[j]} \mathrm{E}\left\{\left(\Pi_{m+j}\right)^{n-k^{\prime}-j} G_{j}\left(1 \mid\left\{\Pi_{m+i}, 0 \leq i \leq n\right\}\right)\right\}$,

which, by (3.1), can be rewritten as (3.10).

Let us now proceed to the general situation with conditional parameters given by (3.7). For fixed values $p_{l}=1-q_{l}$, consider the associated nonstationary Reed-Frost model and let $F_{T}^{c}$ denote the corresponding final number of infections. It is directly seen that Property 2.2 can be extended as follows:

$$
F_{T}^{c,(1)} \leq_{\mathrm{D}} F_{T}^{c} \leq_{\mathrm{D}} F_{T}^{c,(2)},
$$

where $F_{T}^{c,(1)}$ is the final number of infections obtained with parameters

$$
\pi_{l}^{c,(1)}=\mathrm{E}(\varepsilon)+[1-\mathrm{E}\{\varepsilon\}] q_{1} \cdots q_{l}, \quad 1 \leq l \leq m+n,
$$

and $F_{T}^{c,(2)}$ is the final number of infections obtained with parameters

$$
\pi_{l}^{c,(2)}=\left[q_{1}+p_{1} \mathrm{E}\{\varepsilon\}\right] \cdots\left[q_{l}+p_{l} \mathrm{E}\{\varepsilon\}\right] .
$$

Therefore, removing the conditioning on the $P_{l}$, we deduce the following bounds for the law of $F_{T}$.

Property 3.1. Under (3.7), we have $F_{T}^{(1)} \leq_{\mathrm{D}} F_{T} \leq_{\mathrm{D}} F_{T}^{(2)}$, where the law of $F_{T}^{(1)}$ is determined in an all/nothing model with the conditional parameters (3.9) with $\gamma_{1}=\mathrm{E}\{\varepsilon\}$, and the law of $F_{T}^{(2)}$ is determined in a leaky model with the conditional parameters (3.8) with $r=\mathrm{E}\{\varepsilon\}$.

\subsection{Combination with randomized susceptibilities}

The next step is to consider a more general Reed-Frost model that incorporates both randomized infectiousness and susceptibility. In a previous paper, the authors proposed a tentative modelling approach to describe this situation (see Lefèvre and Picard (1999)). As pointed out by Ball (2000), however, the model built does not allow us to account for these two random factors in a completely appropriate way.

In the spirit of Section 2.3, we are going to formulate such a model on the basis of the infection tolerances (see also, for instance, Ball and O'Neill (1999)). Specifically, each infective $l$ now exerts a random power of infection that corresponds to its infectious period $D_{l}$, and any given susceptible is infected as soon as its total absorbed exposure to infection reaches a certain (personal) random threshold distributed as $\xi$; standard independence conditions are assumed to hold. Thus, for fixed values $d_{l}$ of $D_{l}$, the conditional parameters are defined by

$$
\pi_{l}^{c}=\mathrm{P}\left(\xi>d_{1}+\cdots+d_{l}\right), \quad l=1, \ldots, m+n .
$$

In particular, if the $D_{l}$ are constant and equal to $d$, then $\pi_{l}^{c}=\pi_{l}$ for each $l$ and (3.12) reduces to (2.12). 
The law of $F_{T}$ is here provided by the expectation of (2.5) with

$$
\pi_{l}=\mathrm{P}\left(\xi>D_{1}+\cdots+D_{l}\right), \quad 1 \leq l \leq m+n .
$$

This result can be found in Ball and O'Neill (1999, Equation (3.5)).

When $\xi$ has an exponential law with parameter $\beta$, we have $\pi_{l}=\exp \left(-\beta D_{1}\right) \cdots \exp \left(-\beta D_{l}\right)$ for all $l$, meaning that the model reduces to a situation discussed in Section 3.1 with randomized infectiousness only. Let us now suppose that the $D_{l}$ are independent, identically distributed random variables valued on an interval $(0, c)$, and that $\xi$ has a uniform distribution on $(0, \alpha)$, where $\alpha \geq(m+n) c$. Then (3.12) becomes

$$
\pi_{l}^{c}=1-\left(d_{1}+\cdots+d_{l}\right) / \alpha, \quad l=1, \ldots, m+n .
$$

Let $\bar{D}_{l}=\left(D_{1}+\cdots+D_{l}\right) / \alpha, 1 \leq l \leq m+n$. We will show that the distribution of $F_{T}$ is now provided by the explicit formula (3.14), below. Notice that the law (3.14) generalizes (2.14) and can be viewed as a randomized extension of a quasibinomial distribution.

Corollary 3.2. Under (3.13),

$$
\mathrm{P}\left(F_{T}=j\right)=\left(\begin{array}{c}
n \\
j
\end{array}\right) \mathrm{E}\left\{\bar{D}_{m}\left(\bar{D}_{m+j}\right)^{j-1}\left(1-\bar{D}_{m+j}\right)^{n-j}\right\}, \quad j=0, \ldots, n .
$$

Proof. First, inserting (3.13) into (2.5), using (A.4), and taking the expectation with respect to the $D_{l}$ yields

$$
\mathrm{P}\left(F_{T}=j\right)=n_{[j]} \mathrm{E}\left\{\left(1-\bar{D}_{m+j}\right)^{n-j} G_{j}\left(0 \mid\left\{-\bar{D}_{m+i}, 0 \leq i \leq n\right\}\right)\right\}, \quad j=0, \ldots, n .
$$

Let $\bar{D}_{0}^{(m)}=0$ and $\bar{D}_{i}^{(m)}=\left(D_{m+1}+\cdots+D_{m+i}\right) / \alpha, 1 \leq i \leq n$; again by (A.4), (3.15) can then be rewritten as

$$
\mathrm{P}\left(F_{T}=j\right)=n_{[j]} \mathrm{E}\left\{\left(1-\bar{D}_{m}-\bar{D}_{j}^{(m)}\right)^{n-j} G_{j}\left(\bar{D}_{m} \mid\left\{-\bar{D}_{i}^{(m)}, 0 \leq i \leq n\right\}\right)\right\} .
$$

To simplify the expectation in (3.16), we have recourse to Theorem 2.2 of Picard and Lefèvre (2003). More precisely, it is easily seen that the following identity is a direct implication of that theorem. Let $X_{i}, i \geq 1$, be a sequence of nonnegative independent, identically distributed random variables, and denote by $S_{i}=X_{1}+\cdots+X_{i}, i \geq 1$, the associated partial sums, with $S_{0}=0$; then, for any natural number $k$ and any real numbers $x$ and $y$,

$$
\mathrm{E}\left\{\left(y-S_{j}\right)^{k} G_{j}\left(x \mid\left\{-S_{i}, i \in \mathbb{N}\right\}\right)\right\}=\frac{\mathrm{E}\left\{\left(y-S_{j}\right)^{k} x\left(x+S_{j}\right)^{j-1}\right\}}{j !}, \quad j \in \mathbb{N} .
$$

Of course, (3.17) holds also when $x$ and $y$ are replaced by random variables independent of $\left\{-S_{i}, i \in \mathbb{N}\right\}$. Therefore, by applying (3.17) (with $n-j$ substituted for $k, 1-\bar{D}_{m}$ for $y, \bar{D}_{m}$ for $x$, and $\bar{D}_{i}^{(m)}$ for $S_{i}$ ), we deduce that (3.16) reduces to (3.14).

To close, it is worth indicating that the present study can be extended to obtain the joint generating function Laplace transform of the final size and severity of the epidemic. Furthermore, this methodology allows us also to deal with multigroup models involving cross-infections and movements of infectives between groups; see, for instance, Picard and Lefèvre (1990) and Ball and O'Neill (1999). 


\section{Appendix A.}

\section{A.1. Abel-Gontcharoff polynomials}

These polynomials, introduced by Gontcharoff (1937), have been studied (and generalized) by the authors in a series of papers motivated by probabilistic applications; see, for instance, Lefèvre and Picard (1990) and Picard and Lefèvre (1996). Let $U=\left\{u_{i}, i \in \mathbb{N}\right\}$ be a given sequence of real numbers (where, recall, $\mathbb{N}=\{0,1,2, \ldots\}$ ). To $U$ we assign a unique family of Abel-Gontcharoff polynomials $G_{j}(x \mid U), j \in \mathbb{N}$, of degree $j$ in $x$ that are defined recursively by the triangular system of equations

$$
\sum_{j=0}^{k} k_{[j]} u_{j}^{k-j} G_{j}(x \mid U)=x^{k}, \quad k \in \mathbb{N} .
$$

Note that, by (A.1),

$$
G_{0}(x \mid U)=1 \quad \text { and } \quad G_{j}\left(u_{0} \mid U\right)=\delta_{j, 0}, \quad j \in \mathbb{N},
$$

and $G_{j}(x \mid U), j \geq 1$, depends on $U$ only through the elements $\left\{u_{0}, \ldots, u_{j-1}\right\}$. Alternative definitions of the Abel-Gontcharoff polynomials are available (for example, through an integral representation).

Identity (A.1) can be generalized as follows: any polynomial $p(x)$ of degree $k$ admits an Abelian-type expansion with respect to the $G_{j}$, namely

$$
p(x)=\sum_{j=0}^{k} p^{(j)}\left(u_{j}\right) G_{j}(x \mid U),
$$

where $p^{(j)}(x)$ is the $j$ th derivative of $p(x)$. The Abel-Gontcharoff polynomials satisfy various basic properties. For instance, for any reals $a$ and $b$, we have

$$
G_{j}(a x+b \mid a U+b)=a^{j} G_{j}(x \mid U),
$$

where $a U+b$ is the family $\left\{a u_{i}+b, i \in \mathbb{N}\right\}$; for $k=1, \ldots, j$, we have

$$
G_{j}^{(k)}(x \mid U)=G_{j-k}\left(x \mid E^{k} U\right),
$$

where $E^{k} U$ is the family $\left\{u_{k+i}, i \in \mathbb{N}\right\}$; and, if $u_{i}$ is affine in $i$, we have

$$
G_{j}(x \mid U)=\frac{\left(x-u_{0}\right)\left(x-u_{j}\right)^{j-1}}{j !},
$$

which are the classical Abel polynomials. A few other properties used in the paper (but not recalled here) were derived in Lefèvre and Picard (1993) and Picard and Lefèvre (2003).

\section{A.2. A formula equivalent to (2.5)}

In Picard and Lefèvre (1991), the authors studied the model presented in Section 2.3 for which $\pi_{l}=\mathrm{E}\left\{R^{l}\right\}, 1 \leq l \leq m+n, R$ being any random variable valued in $[0,1]$. There it was shown that the law of $F_{T}$ is given by

$$
\mathrm{P}\left(F_{T}=j\right)=n_{[j]} \sum_{k=0}^{j} \frac{1}{k !}\left(\pi_{m+k}\right)^{n-k} H_{n-k}^{(n-j)}(0), \quad j=0, \ldots, n,
$$


where $H_{n-j}^{(n-j)}(0)=1$ and

$$
H_{n-k}^{(n-j)}(0)=-\sum_{l=k+1}^{j} \frac{1}{(l-k) !}\left(\frac{\pi_{m+l}}{\pi_{m+k}}\right)^{n-l} H_{n-l}^{(n-j)}(0), \quad k=0, \ldots, j-1 .
$$

Equation (A.7) is less convenient to apply than (2.5). Let us check that they are equivalent.

First, we will show that (A.8) is equivalent to

$$
H_{n-k}^{(n-j)}(0)=\frac{\left(\pi_{m+j}\right)^{n-j}}{\left(\pi_{m+k}\right)^{n-k}} G_{j-k}\left(0 \mid\left\{\pi_{m+k+i}, 0 \leq i \leq n-k\right\}\right), \quad k=0, \ldots, j .
$$

Let us proceed by induction and suppose that (A.9) holds for $k=l^{\prime}+1, \ldots, j$, say. By inserting (A.9) in (A.8) for $k=l^{\prime}$, we then find that

$$
H_{n-l^{\prime}}^{(n-j)}(0)=-\frac{\left(\pi_{m+j}\right)^{n-j}}{\left(\pi_{m+l^{\prime}}\right)^{n-l^{\prime}}} \sum_{l=l^{\prime}+1}^{j} \frac{\left(\pi_{m+l^{\prime}}\right)^{l-l^{\prime}}}{\left(l-l^{\prime}\right) !} G_{j-l}\left(0 \mid\left\{\pi_{m+l+i}, 0 \leq i \leq n-l\right\}\right),
$$

which, by (A.5), can be re-expressed as

$$
H_{n-l^{\prime}}^{(n-j)}(0)=-\frac{\left(\pi_{m+j}\right)^{n-j}}{\left(\pi_{m+l^{\prime}}\right)^{n-l^{\prime}}} \sum_{l=1}^{j-l^{\prime}} \frac{\left(\pi_{m+l^{\prime}}\right)^{l}}{l !} G_{j-l^{\prime}}^{(l)}\left(0 \mid\left\{\pi_{m+l^{\prime}+i}, 0 \leq i \leq n-l^{\prime}\right\}\right) .
$$

Now, by (A.2), $G_{j-l^{\prime}}\left(\pi_{m+l^{\prime}} \mid\left\{\pi_{m+l^{\prime}+i}, 0 \leq i \leq n-l^{\prime}\right\}\right)=0$ for $j-l^{\prime} \geq 1$. By Taylor expanding this polynomial and using (A.5), we then find that (A.10) reduces to (A.9) for $k=l^{\prime}$, as required.

Second, we may substitute (A.9) into (A.7), to yield

$$
\mathrm{P}\left(F_{T}=j\right)=n_{[j]}\left(\pi_{m+j}\right)^{n-j} \sum_{k=0}^{j} \frac{1}{k !} G_{j-k}\left(0 \mid\left\{\pi_{m+k+i}, 0 \leq i \leq n-k\right\}\right) .
$$

Again by a Taylor expansion, we find that (A.11) is equivalent to (2.5), which is the desired result.

\section{Acknowledgements}

We thank Frank Ball for providing us with the preprint mentioned in the references (Ball (2000)). We are grateful to Sergey Utev for many interesting discussions, especially on the problem of vaccination. We also thank the referee for a careful reading of the paper and useful remarks and suggestions.

\section{References}

Addy, C. L., Longini, I. M. And Haber, M. (1991). A generalized stochastic model for the analysis of infectious disease final size data. Biometrics 47, 961-974.

Andersson, H. ANd Britton, T. (2000). Stochastic Epidemic Models and Their Statistical Analysis (Lecture Notes Statist. 151). Springer, New York.

BALL, F. G. (1986). A unified approach to the distribution of total size and total area under the trajectory of infectives in epidemic models. Adv. Appl. Prob. 18, 289-310. 
BALl, F. G. (2000). Susceptibility sets and the final outcome of stochastic SIR epidemic models. Preprint, School of Mathematical Sciences, The University of Nottingham.

Ball, F. G. ANd O'NeILl, P. (1999). The distribution of general final state random variables for stochastic epidemic models. J. Appl. Prob. 36, 473-491.

Ball, F. G., Mollison, D. and Scalia-Tomba, G. (1997). Epidemics with two levels of mixing. Ann. Appl. Prob. 7, 46-89.

Becker, N. G. And Utev, S. (2002). Protective vaccine efficacy when vaccine response is random. Biometrical J. 44, 29-42.

Consul, P. C. (1974). A simple urn model dependent upon predetermined strategy. Sankhyā B 36, 391-399.

Daley, D. J. AND Gani, J. (1999). Epidemic Modelling: an Introduction. Cambridge University Press.

Gontcharoff, W. (1937). Détermination des Fonctions Entières par Interpolation. Hermann, Paris.

Islam, M. N., O’Shaughnessy, C. D. And Smith, B. (1996). A random graph model for the final-size distribution of household infections. Statist. Medicine 15, 837-843.

LefÈvre, C. (1990). Stochastic epidemic models for SIR infectious diseases: a brief survey of the recent general theory. In Stochastic Processes in Epidemic Theory (Lecture Notes Biomath. 86), eds J.-P. Gabriel, C. Lefèvre and P. Picard, Springer, New York, pp. 1-12.

Lefèvre, C. AND Picard, P. (1990). A nonstandard family of polynomials and the final size distribution of Reed-Frost epidemic processes. Adv. Appl. Prob. 22, 25-48.

Lefèvre, C. AND PiCARD, P. (1993). An unusual stochastic order relation with some applications in sampling and epidemic theory. Adv. Appl. Prob. 25, 61-83.

Lefèvre, C. AND Picard, P. (1999). Abel-Gontcharoff pseudopolynomials and the final outcome of SIR epidemic models. III. Adv. Appl. Prob. 31, 532-550.

Lefèvre, C. And Utev, S. (1996). Comparing sums of exchangeable Bernoulli random variables. J. Appl. Prob. 33, 285-310.

Ludwig, D. (1975). Final size distributions for epidemics. Math. Biosci. 23, 33-46.

Martin-LöF, A. (1986). Symmetric sampling procedures, general epidemic processes and their threshold limit theorems. J. Appl. Prob. 23, 265-282.

PiCARD, P. AND LeFÈVRe, C. (1990). A unified analysis of the final state and severity distribution in collective Reed-Frost epidemic processes. Adv. Appl. Prob. 22, 269-294.

PiCARD, P. AND LefÈvre, C. (1991). The dimension of Reed-Frost epidemic models with randomized susceptibility levels. Math. Biosci. 107, 225-233.

PiCARD, P. AND LefÈvRe, C. (1996). First crossing of basic counting processes with lower non-linear boundaries: a unified approach through pseudopolynomials. I. Adv. Appl. Prob. 28, 853-876.

PiCARD, P. AND LEFÈVRE, C. (2003). On the first meeting or crossing of two independent trajectories for some counting processes. Stoch. Process. Appl. 104, 217-242.

Scalia-Tomba, G. (1985). Asymptotic final size distribution for some chain-binomial processes. Adv. Appl. Prob. 17, 477-495.

Sellke, T. (1983). On the asymptotic distribution of the size of a stochastic epidemic. J. Appl. Prob. 20, $390-394$.

Smith, P. G., Rodrigues, L. C. ANd Fine, P. E. M. (1984). Assessment of the protective efficacy of vaccines against common diseases using case-control and cohort studies. Internat. J. Epidemiology 13, 87-93.

Von Bahr, B. And Martin-Löf, A. (1980). Threshold limit theorems for some epidemic processes. Adv. Appl. Prob. 12, 319-349. 